\title{
Effectiveness of malic acid $1 \%$ in patients with xerostomia induced by antihypertensive drugs
}

\author{
Gerardo Gómez-Moreno ${ }^{1}$, Javier Guardia ${ }^{2}$, Antonio Aguilar-Salvatierra ${ }^{3}$, Maribel Cabrera-Ayala ${ }^{3}$, José- \\ Eduardo Maté-Sánchez de-Val ${ }^{4}$, José-Luis Calvo-Guirado ${ }^{5}$
}

\footnotetext{
${ }^{1}$ Senior Lecturer of Special Care in Dentistry. Professor responsible of Pharmacological Interactions in Dentistry. Faculty of Dentistry, University of Granada, Granada, Spain

${ }^{2}$ Doctor in Dentistry. Collaborator of Pharmacological Interactions in Dentistry. Faculty of Dentistry, University of Granada, Granada, Spain

${ }^{3}$ Degree in Dentistry. Collaborator of Pharmacological Interactions in Dentistry. Faculty of Dentistry, University of Granada, Granada, Spain

${ }^{4}$ Associate Professor Restorative Dentistry. Faculty of Medicine and Dentistry, University of Murcia, Murcia, Spain

${ }^{5}$ Senior Lecturer of General and Implant Dentistry. Faculty of Medicine and Dentistry, University of Murcia, Murcia, Spain
}

Correspondence:

Facultad de Odontología

Campus Universitario de Cartuja

Colegio Máximo s, $n$

E 18071. Granada. Spain

ggomez@ugr.es

\begin{abstract}
Gómez-Moreno G, Guardia J, Aguilar-Salvatierra A, Cabrera-Ayala M, Maté-Sánchez de-Val JE, Calvo-Guirado JL. Effectiveness of malic acid $1 \%$ in patients with xerostomia induced by antihypertensive drugs. Med Oral Patol Oral Cir Bucal. 2013 Jan 1;18 (1):e49-55.

http://www.medicinaoral.com/medoralfree01/v18i1/medoralv18ilp49.pdf
\end{abstract}

Received: $13 / 01 / 2012$ Accepted: 07/06/2012

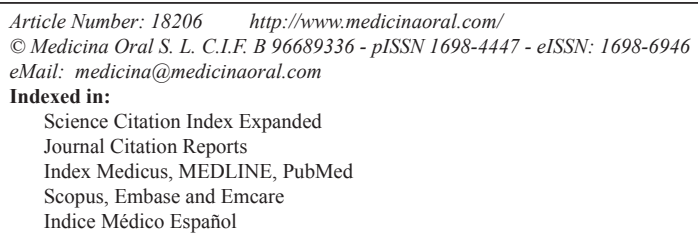

\begin{abstract}
Objectives: Assessing the clinical effectiveness of a topical sialogogue on spray (malic acid, 1\%) in the treatment of xerostomia induced by antihypertensive drugs.

Study Design: This research has been carried out through a randomized double-blind clinical trial. 45 patients suffering from hypertensive drugs-induced xerostomia were divided into 2 groups: the first group ( 25 patients) received a topical sialogogue on spray (malic acid, 1\%) whereas the second group (20 patients) received a placebo. Both of them were administered on demand for 2 weeks. Dry Mouth Questionnaire (DMQ) was used in order to evaluate xerostomia levels before and after product/placebo application. Unstimulated and stimulated salivary flows rates, before and after application, were measured. All the statistical analyses were performed by using SPSS software v17.0. Different DMQ scores at the earliest and final stage of the trial were analysed by using Mann-Whitney $U$ test, whereas Student's T-test was used to analyse salivary flows. Critical $\mathrm{p}$-value was established at $\mathrm{p}<0.05$.

Results: DMQ scores increased significantly (clinical recovery) from 1.21 to 3.36 points $(\mathrm{p}<0.05)$ after malic acid (1\%) application whereas DMQ scores increased from 1.18 to 1.34 points ( $\mathrm{p}>0.05)$ after placebo application. After two weeks of treatment with malic acid, unstimulated salivary flow increased from 0.17 to $0.242 \mathrm{~mL} / \mathrm{min}$ whereas
\end{abstract}


the stimulated one increased from 0.66 to $0.92 \mathrm{~mL} / \mathrm{min}(\mathrm{p}<0.05)$. After placebo application unstimulated flow ranged from 0.152 to $0.146 \mathrm{~mL} / \mathrm{min}$ and stimulated flow increased from 0.67 to $0.70 \mathrm{~mL} / \mathrm{min}(\mathrm{p}>0.05)$.

Conclusions: Malic acid 1\% spray improved antihypertensive-induced xerostomia and stimulated the production of saliva.

Key words: Xerostomia, hyposialia, malic acid, antihypertensive drugs.

\section{Introduction}

Xerostomia is a subjective complaint of dry mouth, and it is usually caused by a decreased salivary flow or by changes in the biochemical composition of saliva. Patients suffering from xerostomia usually complain about difficulties when chewing, swallowing or even speaking, particularly those with dental prosthesis. Whereas xerostomia is a subjective concept, hyposalivation makes reference to a decreased salivary flow and it is, therefore, an objective and measurable variable (1-3).

Hyposalivation is considered to appear when salivary flow rates are under $0.1-0.2 \mathrm{~mL} / \mathrm{min}$ (unstimulated) or $0.7 \mathrm{~mL} / \mathrm{min}$ (stimulated). Xerostomia is often associated with hyposalivation, but not always. It is widely accepted that a salivary flow rate reduction of around $50 \%$ implies the manifestation of signs and symptoms of xerostomia $(4,5)$. However, some cases have been described in patients with a normal salivary flow rate. Furthermore, other patients with a decreased salivary flow rates (generally fewer than $50 \%$ if compared to normal levels) have not complaint about oral problems.

The most important aetiological factors related to xerostomia are: head and neck radiotherapy, some systemic conditions (primary or secondary Sjögren syndrome, stress, diabetes, clinical depression ) and also the intake of certain drugs $(1,6,7)$. More than 500 drugs (8), including 42 different pharmacological groups (9) can cause xerostomia as a side effect. Drugs with the most intense xerostomizing effect are: -those with a direct impact on the autonomic nervous system, which regulates gland secretions (anticholinergic agents; adrenergic blockers); - those with an indirect impact on the central nervous system (antipsychotic drugs, anxiolytic agents); - those which increase the excretion of liquids, particularly diuretics. In addition to this, some psychological factors, such as stress, anxiety or depressive conditions are also related to xerostomia. However, hiposalivation is normally associated with drug-induced xerostomia, whereas this association is not usual in the case of xerostomia related to psychological conditions (10).

Drugs most commonly associated with xerostomia are: -antidepressants (particularly tricyclic antidepressants) (11); - Selective Serotonin Reuptake Inhibitors (SSRIs), particularly when combined with benzodiazepines (12); -Diuretics, antihypertensive drugs and angiotensinconverting enzyme inhibitors (ACE inhibitors), -oral hypoglycemiants,-acetylsalicylic acid (ASA), -iron sup- plements. Let us not forget that drugs with the most intense xerostomizing effect are also the most widely and frequently used (treatment of metal disorders and cardiovascular diseases) (13). This fact is useful to explain the important prevalence among adults older than $65(20-46 \%)(6,14,15)$. Such prevalence is caused by 3 important factors: accumulation of systemic conditions, polymedication and the progressive decline of salivary gland parenchyma.

There is a wide range of therapies in the treatment of xerostomia (sialogogues, salivary substitutes, general measures...), although the efficiency of many of them is controversial $(1,6,7)$. Abundant literature has described malic and citric acid as salivary stimulants, although they were dropped out because their demineralizing effect on the tooth enamel $(16,17)$. Nevertheless, recent researches have reported a decrease of this demineralizing potential of malic acid, if used at an appropriate concentration (4.7\%) and combined with xylitol and fluorides (18).

Assessing the clinical efficiency of a topical sialogogue on spray (malic acid 1\%), combined with xylitol and fluoride, in the treatment of xerostomia induced by antihypertensive drugs for 2 weeks has been the main objective of this research.

\section{Material and Methods}

-Patients recruitment and intervention

This randomized double-blind clinical trial has been approved by the University of Granada Ethics Committee (Spain). Randomization was done following the guidelines of The Consort Statement (http://www. consort-statement.org/consort-statement/). The sample size calculation was performed from the standard deviation of the main variable (DMQ). 45 participants with xerostomia caused by the chronic admnistration of antihypertensive drugs, were recruited at the Faculty of Dentistry of the University of Granada (Spain) and also at the Faculty of Medicine and Dentistry of the University of Murcia (Spain). No participants left the trial.

-Inclusion Criteria

-Subjects under antihypertensive treatment (longer than one month) with xerostomia.

-Exclusion Criteria

-History of head and neck radiotherapy, Sjögren's syndrome and related autoimmune diseases (rheumatoid 
arthritis, polyarthritis nodosa, systemic sclerosis or lupus erythematosus).

-Subjects with diabetes and/or oral hypoglycemic therapy. -Chronic alcoholic subjects

The 45 patients suffering from hypertensive drugsinduced xerostomia were divided into 2 groups: the first group (25 patients) received a topical sialogogue on spray (malic acid, 1\%) whereas the second group (20 patients) received a placebo. 16 patients were being treated with diuretics, 14 with angiotensin-converting enzyme inhibitors (ACE inhibitors), 7 with non-selective beta-blockers, and 8 with angiotensin II receptor antagonists.

The randomized distribution was designed by using the websitehttp://www.randomization.com, and obtaining a randomization plan, which assigned participants to a "test group" (to be treated with $1 \%$ malic acid on spray) or to a "placebo group" (to be treated with a spray with no malic acid). This randomization plan was delivered to a person not related to the project in order to prevent both participants and observers from identifying the product.

Once the patients had signed the informed consent form and anamnesis had been performed, the following question was asked to every patient: "How often do you feel dry mouth?" Available answers were: "never", "some- times", "usually" or "always". Those who answered "usually" or "always" were considered as suffering from xerostomia.

Our clinical intervention was based on the application of a topical sialogogue, containing 1\% malic acid (Xeros Dentaid spray $($, Dentaid, Barcelona, Spain) for 2 weeks in an experimental group of 25 patients, whereas a control group of 20 patients was given a placebo with the same presentation and composition (excepting malic acid). Product/placebo was presented without any brand name, and they were administered on demand, with a maximum of 8 doses per day.

Dry Mouth Questionnaire (DMQ)

DMQ, developed by Vissink et al. (19), Gravenmade et al. (20), van der Reijden et al. (21) y Regelink et al. (22) (Table 1), was used in order to obtain subjective information about the severity of xerostomia before and after treatment with malic acid/placebo. Every participant had to answer an initial questionnaire (DMQ 1) about the symptoms related to oral dryness, and received a spray (malic acid or placebo). After 2 weeks of treatment, patients had to answer again DMQ 1, and also a new questionnaire (DMQ 2) about the efficiency of the treatment.

DMQ 1 was used to assess the initial severity of oral dryness, particularly its impact on oral function: prob-

Table 1. Dry Mouth Questionnaire (DMQ).

\begin{tabular}{|c|c|}
\hline \multicolumn{2}{|c|}{ DMQ 1} \\
\hline \multicolumn{2}{|c|}{ The questions have to be completed both at baseline and after two weeks of use of the agent } \\
\hline Question & Response (scale 0-4) \\
\hline How dry is your mouth? & VERY DRY-NOT DRY \\
\hline Are you suffering from oral dryness during daytime? & VERY SEVERE-NEVER \\
\hline Are you suffering from oral dryness at night? & VERY SEVERE-NEVER \\
\hline Do you have a nasty taste in your mouth? & VERY SEVERE-NEVER \\
\hline Is sleeping impeded? & VERY SEVERE-NEVER \\
\hline Is swallowing impeded? & VERY SEVERE-NEVER \\
\hline Is eating impeded? & VERY SEVERE-NEVER \\
\hline \multicolumn{2}{|c|}{ DMQ 2} \\
\hline \multicolumn{2}{|c|}{ Questions have to be completed after two weeks of use of the agent } \\
\hline Question & Response (scale 0-4) \\
\hline How frequently do you apply the substitute? & ..... TIMES PER DAY \\
\hline For how long is your mouth moist after applying the $\mathrm{s}$ & ...... MIN \\
\hline \multicolumn{2}{|c|}{ Is the extent of oral dryness reduced when applying the substitute? HIGHLY-NOT REDUCED } \\
\hline How dry is your mouth when applying the substitute? & VERY SEVERE-NOT DRY \\
\hline How do you appreciate the taste of the substitute? & VERY PALATABLE-NASTY \\
\hline \multicolumn{2}{|l|}{ What complaints are reduced when using the substitute? } \\
\hline Dryness during daytime & HIGHLY-NOT REDUCED \\
\hline Dryness at night & HIGHLY-NOT REDUCED \\
\hline Burning mouth & HIGHLY-NOT REDUCED \\
\hline Nasty taste & HIGHLY-NOT REDUCED \\
\hline Sleeping difficulties & HIGHLY-NOT REDUCED \\
\hline Difficulties with speech & HIGHLY-NOT REDUCED \\
\hline Difficulties with swallowing & HIGHLY-NOT REDUCED \\
\hline Difficulties with eating & HIGHLY-NOT REDUCED \\
\hline
\end{tabular}


lems when chewing, swallowing, speaking and impact on daily life.

DMQ 1 used a 0-to-4 rating scale where $0=$ "very dry" and $4=$ "not dry at all". After 2 weeks of treatment, DMQ 1 was repeated (Table 1). On the other hand, DMQ 2 was designed to assess the impact of the spray on the symptoms of xerostomia, and was also based on a 0-to-4 rating scale where $0=$ frequent restriction of oral function and $4=$ no restriction of oral function/ no oral dryness feeling. Application frequency and retention time inside the oral cavity were also registered (Table 1).

-Sialometries

As secondary measures, both unstimulated and stimulated salivary flow rates were assessed in all patients. Unstimulated salivary flow rate was obtained through the collection of saliva every 30 seconds during $15 \mathrm{mi}$ nutes. Saliva was collected on $20 \mathrm{~mL}$ plastic containers, which were pre-weighted $(0.001 \mathrm{~g})$ by using a precision scale (Cobos M-150, Cobos, Barcelona, Spain). Measures were expressed as $\mathrm{mL} / \mathrm{min}$. As far as stimulated flow rate is concerned, it was obtained by chewing a $1 \mathrm{~g}$ piece of paraffin during 6 minutes. Saliva collected during the first minute was discarded, and then collected on containers every 30 seconds. DMQ and sialometries were always performed from 09:00 a.m. to 11:00 a.m. in order to avoid any circadian variation. Prior to intervention, patients were told not to eat, drink, smoke or brush their teeth from one hour before their visit.

-Statistical analysis

All of the analyses were performed by using SPSS software v17.0 (SPSS Inc. Chicago, IL, USA). The main purpose was to contrast different DMQ scores at the earliest and final stage of the clinical trial by using the Mann-Whitney U test. Student's T-test was used to analyse both unstimulated and stimulated salivary flow rates. Critical $\mathrm{p}$-value was established at $\mathrm{p}<0.05$.

\section{Results}

Table 2 shows the results of our clinical trial in relation to age, gender, DMQ score (oral dryness feeling), number of applications and duration of sialogogue action. No relevant statistical differences were found as far as gender is concerned. DMQ scores related to dry mouth feeling increased significantly (therefore suggesting a clinical recovery) from $1.21 \pm 0.14$ points to $3.36 \pm 0.17$ points $(p<0.05)$ after two weeks treatment with malic acid, whereas in the control group DMQ scores increased from $1.18 \pm 0.12$ points to $1.34 \pm 0.09$ points $(\mathrm{p}>0.05) .92 \%$ of the patients treated with malic acid experienced some clinical recovery, in contrast to just $15 \%$ of the patients treated with placebo. Moreover, no patient in the experimental group experienced a decline of the initial condition, whereas 3 members of the control group did it. Patients belonging to the experimental group used the product $3.71 \pm 1.32$ times per day,
Table 2. Age, gender, DMQ score (severity of xerostomia) and length of effect of the participants. Average \pm standard deviation.

\begin{tabular}{|l|c|c|}
\hline \multicolumn{1}{|c|}{ VARIABLES } & $\begin{array}{c}\text { EXPERIMENTAL } \\
\text { GROUP }\end{array}$ & $\begin{array}{c}\text { CONTROL } \\
\text { GROUP }\end{array}$ \\
\hline Sample size & 25 & 20 \\
\hline Age (years) & $54,3 \pm 4,3$ & $51,8 \pm 3,4$ \\
\hline Gender & 11 & \\
\hline \multicolumn{1}{|c|}{ Male } & 14 & 11 \\
\hline Female & & \\
\hline DMQ score & $1,21 \pm 0,14$ & $1,18 \pm 0,12$ \\
\hline 0 weeks & $3,36 \pm 0,17^{*}$ & $1,34 \pm 0,09$ \\
\hline 2 weeks & $3,71 \pm 1.32^{*}$ & $6,75 \pm 1,21$ \\
\hline Number of applications & $29,6 \pm 1,25^{*}$ & $5,80 \pm 1,10$ \\
\hline Length of Effect (minutes) & & \\
$* \mathrm{p}<0,05$ & &
\end{tabular}

whereas those belonging to the control group used the placebo $6.75 \pm 1.21$ times per day.

Regarding salivary flow rates, they improved after treatment with $1 \%$ malic acid. After 2 weeks of treatment with the product, unstimulated salivary flow rate increased significantly from $0.170 \mathrm{~mL} / \mathrm{min}$ to $0.242 \mathrm{~mL} /$ $\min (\mathrm{p}<0.05)$, whereas patients treated with placebo ranged from $0.152 \mathrm{~mL} / \mathrm{min}$ to $0.146 \mathrm{~mL} / \mathrm{min}(\mathrm{p}>0.05)$ (Fig. 1). As far as stimulated salivary flow rates are concerned, patients treated with malic acid experienced a significant average increase from $0.660 \mathrm{~mL} / \mathrm{min}$ to $0.920 \mathrm{~mL} / \mathrm{min}$ after 2 weeks treatment $(\mathrm{p}<0.05)$; whereas those patients treated with placebo experienced an average increase from $0.67 \mathrm{~mL} / \mathrm{min}$ to $0.70 \mathrm{~mL} / \mathrm{min}$ after 2 weeks ( $>0.05)$ (Fig. 2).

\section{Discussion}

The most important aetiological factor related to xerostomia is the intake of drugs. The interruption of the treatment or substitution of these drugs could increase the salivary flow rate to the level previous to treatment. Nevertheless, this practice involves a risk for the health of the patient, as it would improve oral dryness but would also aggravate the previous condition (in our research, this previous condition is arterial hypertension).

Xerostomia induced by hypertensive drugs is reversible, since even with this condition the salivary glands are sufficiently functional. Consequently, they can be treated with salivary stimulants (sialogogues). Treatment with systemic sialogogues with anticholinesterasic and cholinergic action represents an efficient therapeutic option, although they are usually dropped out because of the quantitative and qualitative importance of their side effects. In 


\section{UNSTIMULATED SALIVARY FLOW RATE}

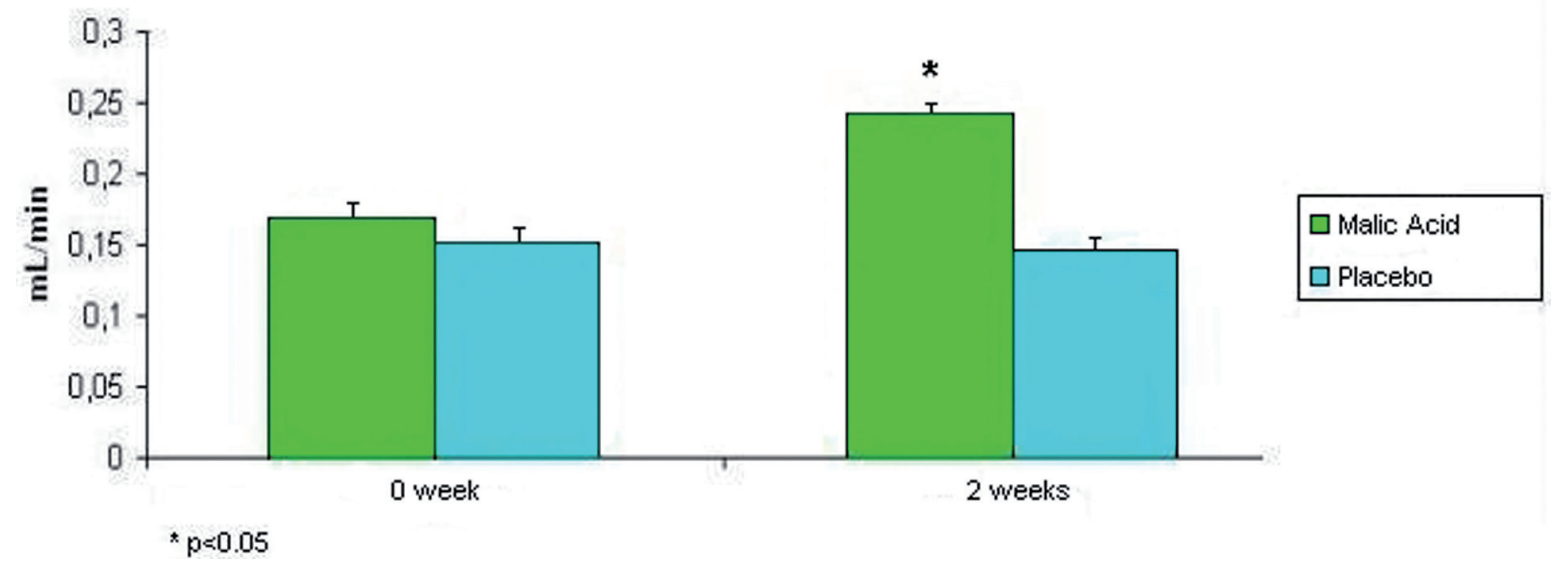

Fig. 1. Average and standard deviation of unstimulated salivary flow rates of the participants (at the beginning and after two weeks of treatment).

\section{STIMULATED SALIVARY FLOW RATE}

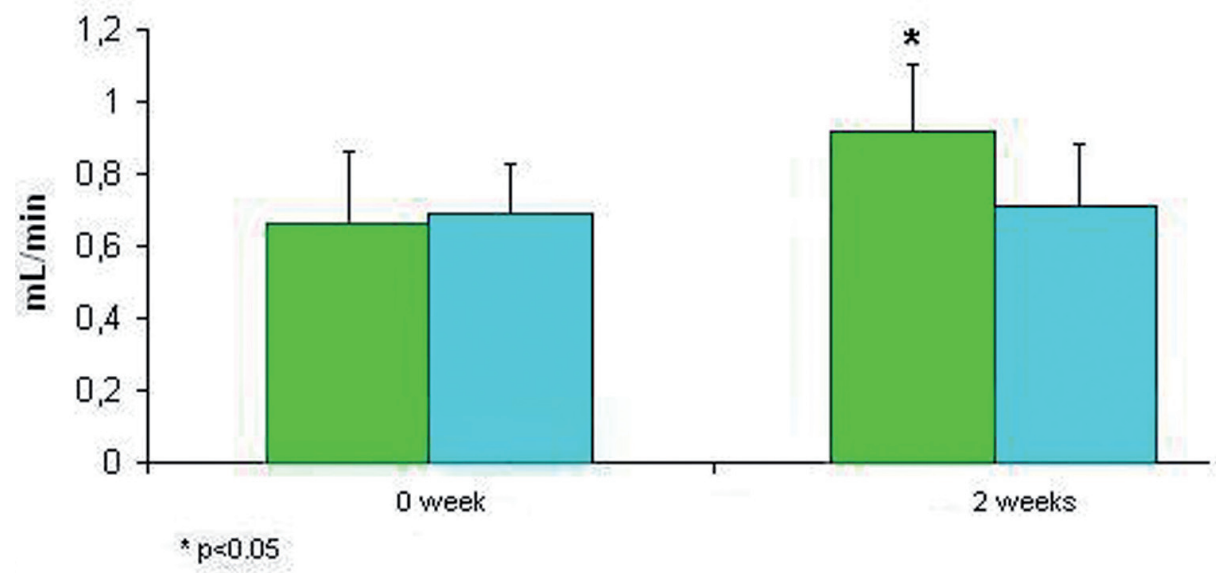

$\square$ Malic Acid

$\square$ Placebo

Fig. 2. Average and standar deviation of stimulated salivary flow rates of the participants (at the beginning and after two weeks of treatment).

this context, the application of topical sialogogues can be a useful alternative in the treatment of reversible xerostomia induced by drugs, as in spite of the fact that their therapeutic effects are more transitory and less lengthy; their side effects are also less powerful (23).

The application of topical sialogogues containing acids in the treatment of xerostomia is not recent. However, continuous application of substances such as citric acid (24) has been related to an increased risk of caries, as a consequence of the erosive action of these agents over the dentin. Similarly, the use of chewing gums containing $0.06 \mathrm{mg}$ of ascorbic or malic acid combined with important quantities of sorbitol and mannitol (16) can cause similar negative effects.

All of these products were dropped out because of their demineralising effect on the human dentin, effect not only caused by the high doses of acidic products, but also by the product presentation (chewable products), which allows a lengthy contact with the dental surfaces. In order to solve this, presentation in spray format allows a fast and direct contact with the oral mucosa, and this fact, if combined with a suitable concentration (as the stimulant effect on saliva production is not altered by it), could reduce the demineralising potential of these substances $(25,26,18)$.

According to the researches carried out by da Mata et al. (18) the use of $4.7 \%$ malic acid on spray (combined with fluorides and xylitol), on 60 healthy subjects induced a significant drop of salivary $\mathrm{pH}$ levels, which recovered 20 minutes later. Nevertheless, when this acidic 
salivary stimulant contains xylitol/fluorides, the subsequent decrease in the salivary $\mathrm{pH}$ level never reaches a score lower than 5.5 (hydroxyapatite critical level) (18). Thus, combination of malic acid with xylitol/fluorides on spray seems to be a safe option as topical sialogogue (9). According to the results of our clinical research, the use of malic acid as a salivary stimulant, if combined with xylitol and fluorides, can be a valid option in the treatment of mild and reversible xerostomia induced by antihypertensive drugs. Malic acid acts as a substance capable of generating a sour taste gustatory stimulus. Its mechanism of action is linked to dissociation of malic acid in $\mathrm{H}+$, which they join water to become hydronium ions $\left(\mathrm{H}_{3} \mathrm{O}^{+}\right)$; this action generates a stimulation of salivary secretion to dilute the concentration of acids in the oral cavity. Xylitol and fluorides do not stimulate saliva but they reduce erosion and cariogenic potential.

When used as a topical sialogogue, this product provides a short term effect increasing salivary flow rates immediately and subjects in this clinical trial reported a feeling of increase saliva production for an average period of 29 minutes (in contrast to an average period of 5 minutes in the control group). These results are in line with the results obtained by da Mata et al. (18).

Salivary flow rates became normal 20-30 minutes after application, and patients did not feel the need for a new application for around 6 hours time, so 3 or 4 applications per day were enough. In contrast, patients treated with placebo used it 6 or 7 times per day.

Among the different available questionnaires to evaluate the severity of oral dryness, we chose the DMQ (1922), since it is an easy and fast method to assess the efficiency of the product. In addition to this, its 0-to-4 scale can be easily replaced by a Visual Analogue Scale (VAS) of $10 \mathrm{~cm}$. Therefore, DMQ has been a valid and useful tool for the carrying out of our clinical trial.

The results of our randomized clinical trial clearly show a significant increase of salivary flow rates, both unstimulated and stimulated, after the treatment with $1 \%$ malic acid on spray. These results are in accordance with those obtained by da Mata et al. (18), although we must point out that in that research, the subjects were healthy individuals who were not suffering from xerostomia. No additional researches or studies have focused on the assessment of the efficiency of malic acid in the treatment of xerostomia, so our clinical trial is one of the first ones dealing with this topic.

As far as subjective improvement of xerostomia is concerned, there are not similar papers to compare the results of our trial, as research in this field has been neglected because of the caries-inducing role of acidic substances when they are not properly formulated. In this sense, our clinical trial is a pioneering work in the field of treatment of xerostomia induced by drugs with topical acidic salivary stimulants.
The results of our clinical trial show that the use of $1 \%$ malic acid on spray causes an improvement of dry mouth feeling, and stimulates saliva production. Consequently, $1 \%$ malic acid may be an effective treatment of antihypertensive-induced xerostomia.

\section{References}

1. Cassolato SF, Turnbull RS. Xerostomia: clinical aspects and treatment. Gerodontology. 2003;20:64-77.

2. Navazesh M, Christensen C, Brightman V. Clinical criteria for the diagnosis of salivaly gland hypofunction. J Dent Res. 1992;71:1363-9.

3. Mittal S, Bansal V, Garg S, Atreja G, Bansal S. The diagnostic role of Saliva -A Review. J Clin Exp Dent. 2011;3:e314-20.

4. Dawes C. Physiological factors affecting salivary flow rate, oral sugar clearance, and the sensation of dry mouth in man. J Dent Res. 1987;66:648-53

5. Wolff MS, Kleinberg I. The effect of ammonium glycopyrrolate (Robinul)-induced xerostomia on oral mucosal wetness and flow of gingival crevicular fluid in humans. Arch Oral Biol. 1999;44:97-102.

6. Gupta A, Epstein JB, Sroussi H. Hyposalivation in elderly patients. J Can Dent Assoc. 2006;72:841-6.

7. Silvestre-Donat FJ, Miralles-Jorda L, Martinez-Mihi V. Protocol for the clinical management of dry mouth. Med Oral. 2004;9:273-9.

8. Scully C, Bagan JV. Adverse drug reactions in the orofacial region. Crit Rev Oral Biol Med. 2004;15:221-39.

9. Martín-Piedra MA, Gómez-Moreno G, Herrera D, Aguilar-Salvatierra A. Effectiveness of a recent topical sialogogue in the management of drug-induced xerostomia. J Clin Exp Dent. 2011;3:e268-73.

10. Bergdahl M, Bergdahl J. Low unstimulated salivary flow and subjective oral dryness: association with medication, anxiety, depression, and stress. J Dent Res. 2000;79:1652-8.

11. Murray Thomson W, Chalmers JM, John Spencer A, Slade GD, Carter KD. A longitudinal study of medication exposure and xerostomia among older people. Gerodontology. 2006;23:205-13.

12. de Almeida Pdel V, Grégio AM, Brancher JA, Ignácio SA, Machado MA, de Lima AA, et al. Effects of antidepressants and benzodiazepines on stimulated salivary flow rate and biochemistry composition of the saliva. Oral Surg Oral Med Oral Pathol Oral Radiol Endod. 2008;106:58-65.

13. Thomson WM, Chalmers JM, Spencer AJ, Williams SM. The Xerostomia Inventory: a multi-item approach to measuring dry mouth. Community Dent Health. 1999;16:12-7.

14. Locker D. Subjective reports of oral dryness in an older adult population. Community Dent Oral Epidemiol. 1993;21:165-8.

15. Narhi TO. Prevalence of subjective feelings of dry mouth in the elderly. J Dent Res. 1994;73:20-5.

16. Anneroth G, Nordenram G, Bengtsson S. Effect of saliva stimulants (Hybrin and malic acid) on cervical root surfaces in vitro. Scand J Dent Res. 1980;88:214-8.

17. Davies AN. A comparison of artificial saliva and chewing gum in the management of xerostomia in patients with advanced cancer. Palliat Med. 2000;14:197-203.

18. da Mata AD, da Silva Marques DN, Silveira JM, Marques JR, de Melo Campos Felino ET, Guilherme NF. Effects of gustatory stimulants of salivary secretion on salivary $\mathrm{pH}$ and flow: a randomized controlled trial. Oral Dis. 2009;15:220-8.

19. Vissink A, Schaub RM, van Rijn LJ, Gravenmade EJ, Panders AK, Vermey A. The efficacy of mucin-containing artificial saliva in alleviating symptoms of xerostomia. Gerodontology. 1987;6:95-101. 20. Gravenmade EJ, Vissink A. Mucin-containing lozenges in the treatment of intraoral problems associated with Sjögren's syndrome. A double-blind crossover study in 42 patients. Oral Surg Oral Med Oral Pathol. 1993;75:466-71.

21. van der Reijden WA, van der Kwaak H, Vissink A, Veerman EC, Amerongen AV. Treatment of xerostomia with polymer-based saliva 
substitutes in patients with Sjögren's syndrome. Arthritis Rheum. 1996;39:57-63.

22. Regelink G, Vissink A, Reintsema H, Nauta JM. Efficacy of a synthetic polymer saliva substitute in reducing oral complaints of patients suffering from irradiation-induced xerostomia. Quintessence Int. 1998;29:383-8.

23. Navazesh M, Christensen CM. A comparison of whole mouth resting and stimulated salivary measurement procedures. J Dent Res. 1982;61:1158-62.

24. Gambon DL, Brand HS, Nieuw Amerongen AV. The erosive potential of candy sprays. Br Dent J. 2009;206:530-1.

25. Chunmuang S, Jitpukdeebodintra S, Chuenarrom C, Benjakul P. Effect of xylitol and fluoride on enamel erosion in vitro. J Oral Sci. 2007:49:293-7.

26. Hove LH, Holme B, Young A, Tveit AB. The protective effect of TiF4, SnF2 and NaF against erosion-like lesions in situ. Caries Res. 2008;42:68-72.

Acknowledgments

This project has been supported by: Proyecto de Investigación FIS

PI10/00932, Ministerio de Ciencia e Innovación, Instituto de Salud Carlos III (Spain) and Proyecto OTRI CNT-2856 in cooperation with the University of Granada and Dentaid S.L. (Barcelona, Spain), within the framework of the research group "CTS-654, Pharmacological Research in Dentistry", sponsored by the Regional Government of Andalucía (Spain). 